\title{
Concise Commentary: Armchair Science Redux-How Advanced In Silico Analysis Revealed Novel Insights into the Pathogenesis of Acute Pancreatitis
}

\author{
Calen A. Steiner ${ }^{1}$. Shrinivas Bishu ${ }^{1}$
}

Published online: 29 January 2020

(c) Springer Science+Business Media, LLC, part of Springer Nature 2020

As the technology available to sequence genetic material becomes increasingly refined and affordable, so too does the opportunity to apply it to the diagnosis and prognosis of human disease. Modern genetic sequencing methods, including RNA sequencing, chromatin immunoprecipitation sequencing, and single-cell sequencing now offer deep views of the genetic state of organisms. This rapid technological expansion has produced an abundance of data, which when combined with modern computing power and statistical techniques have given rise to modern bioinformatics. These advances offer the enticing possibility of advancing understanding of human health and disease, including identifying novel diagnostic, prognostic, and treatment pathways, but are not without their challenges, such as analysis of these enormous data sets so as to make them useful to medicine.

This is the challenge accepted by Fan et al. [1] in their study "Identification of acute pancreatitis related genes and pathways by integrated bioinformatics analysis" published in this issue of Digestive Diseases and Sciences. In this paper, Fan et al. utilize widely available databases and thoughtful bioinformatics techniques in order to increase understanding of the diagnosis, prognosis, and pathogenesis of acute pancreatitis (AP) and complications thereof. Through multiple techniques, they have drawn from widely available databases in order to transform a large amount of raw data into several usable insights about pancreatitis.

Using the publicly available Gene Expression Omnibus (GEO) database, they were able to identify two relevant mRNA datasets from well-characterized murine models for analysis [2]. Although there are weaknesses in the use of animal models to study human disease, these data do

Shrinivas Bishu

bishus@med.umich.edu

1 Division of Gastroenterology and Hepatology, Department of Internal Medicine, University of Michigan, Ann Arbor, USA constitute a readily available and reasonable starting point from which to launch a bioinformatics-based study of pancreatitis. Using these datasets, the authors identified differentially expressed genes (DEG) within and overlapping DEGs between the datasets in order to construct a list of acute pancreatitis-related DEG. The authors then functionally annotated the genes using standard methods (gene ontology and the Kyoto Encyclopedia of Genes and Genomes) in order to identify involved biological processes. They then identified gene-protein and protein-protein interactions using network analysis coupled with publically available methods to construct a hub-node network, subsequently validating protein expression in the human pancreas using the (again publically available) Human Protein Atlas.

The authors then analyzed the interactions between the proteins encoded by these genes using the Search Tool for the Retrieval of Interacting Genes (STRING) and conducted a protein-protein interaction (PPI) network of the identified protein interactions using Cytoscape software. This network featured 65 hub genes and identified five nodes with a high degree of interaction, which included EGFR, CDH1, ACTB, CD44, and VCL. Cytotype MCODE analysis then identified a total of 11 nodes, with all five of the aforementioned nodes being identified. This portion of the analysis utilized complicated network analysis in order to find potentially important interactions of genes and their products. These nodes of interest were then fed into GO and KEGG database that found similar associations as the above DEG analysis, namely that these interactions were involved in similar processes such as cytoskeletal regulation, adhesion, and cancer pathways. Furthermore, they explored genetic regulation of transcripts by associating gene transcription factor pairs at hub genes and ended by validating this work by showing that high expression of hub genes predicts poor survival in pancreatic cancer.

Thus, this study elegantly shows that murine data can be translated in order to gain insights into human disease, thus 
impressively traversing the "bench-to-bedside" paradigm entirely in silico. This research serves as a blueprint for the use of publicly available data and readily available software in order to gain important insights into an important disease that can be used to generated novel and testable hypotheses.

\section{References}

1. Fan L, Hui X, Mao Y, et al. Identification of acute pancreatitis related genes and pathways by integrated bioinformatics analysis.
Dig Dis Sci. (Epub ahead of print). https://doi.org/10.1007/s1062 0-019-05928-5.

2. Hyun JJ, Lee HS. Experimental models of pancreatitis. Clin Endosc. 2014;47:212-216.

Publisher's Note Springer Nature remains neutral with regard to jurisdictional claims in published maps and institutional affiliations. 